\title{
New synchronization method of arbitrary different radio frequencies in accelerators
}

\author{
Y. Kawashima, T. Asaka, and T. Takashima \\ SPring-8, 1-1-1 Kouto Mikazuki-cho Sayo-gun, Hyogo 679-5198, Japan
}

(Received 4 June 2001; published 8 August 2001)

\begin{abstract}
In electron accelerator facilities, radio frequencies for a linac accelerator and a circular accelerator including a booster synchrotron ring and a storage ring are completely different. In order to improve the beam transmission efficiency from a linac to a circular accelerator, and to make the beam intensity constant, both rf's are generally synchronized. This method is well known and uses a common subharmonic frequency of around $10 \mathrm{MHz}$. The rf's used are generated by using a frequency multiplier from the common frequency, and they are continuously generated. We paid attention to the fact that the electron beam from a linac accelerator is always pulsed, and the rf of the linac requires only a moment during beam emission and acceleration. Taking this fact into account, we invented a new synchronization method for the rf's of both the linac and the circular accelerators. The new method is explained by describing a concrete example at the synchrotron radiation facility, SPring-8 (Super Photon ring $8 \mathrm{GeV}$ ). A $2856 \mathrm{MHz}$ rf for the linac is generated by $508.58 \mathrm{MHz}$ rf of the storage ring for a short duration of about $290 \mu \mathrm{s}$. Thus, the phase difference between the two rf's is automatically fixed. This method can be applied to any combination of arbitrary different rf's.
\end{abstract}

DOI: 10.1103/PhysRevSTAB.4.082001

PACS numbers: 41.75.Fr

SPring-8 (Super Photon ring $8 \mathrm{GeV}$ ) [1] is a synchrotron radiation facility consisting of three accelerators: a linac, a booster synchrotron, and a storage ring. The linac has a $2856 \mathrm{MHz}$ radio frequency. A $508.58 \mathrm{MHz}$ rf is used in both the booster synchrotron and the storage rings. The phase between the 508.58 and $2856 \mathrm{MHz}$ rf's is not locked. The time width of a beam emitted from the electron gun in the linac is formed by the time width of high voltage generated by a grid pulser produced by Kentech Instruments. We can currently choose a $1 \mathrm{~ns}$ pulse or a $40 \mathrm{~ns}$ pulse as the pulse width of the grid pulser. To achieve a much better single-bunch mode operation required by users in the storage ring, we will exchange the $1 \mathrm{~ns}$ grid pulser with another pulser with a shorter pulse width of $250 \mathrm{ps}$ FWHM [2]. In the electron beam bunching section, two prebunchers consisting of a reentrant-type single-cell cavity are placed behind the electron gun high-voltage deck, and a main buncher composed of a 13-cell cavity is installed behind them [3]. Each beam-bunching section also uses a $2856 \mathrm{MHz}$ rf. On the other hand, the gun trigger signal is generated by a $508.58 \mathrm{MHz}$ rf. Thus, an electron beam with a time width of 250 ps FWHM emitted through the grid enters into the first prebuncher, and the beam intensity changes depending on the phase difference between the gun trigger signal and the $2856 \mathrm{MHz}$ rf. In fact, the beam intensity from the linac to the booster synchrotron changes wildly shot by shot. In order to keep the electron beam intensity constant even in the case of a grid pulser with a 250 ps time width, the phase difference between both the 508.58 and $2856 \mathrm{MHz}$ rf's should be fixed. These two rf's should be synchronized. The best way to synchronize the two rf's is to adopt an rf for a circular accelerator and an rf for a linac accelerator multiplied by an arbitrary integer. Unfortunately, we first chose klystrons as amplifiers for the two rf's, and the rf's were automatically chosen to fit the klystrons. Consequently, it became very hard to synchronize the two rf's.

At the B-factory at KEK (High Energy Accelerator Research Organization) in Japan, they recently generated $2856 \mathrm{MHz}$ for the linac and $508.887 \mathrm{MHz}$, instead of $508.58 \mathrm{MHz}$, for the storage ring using the common subharmonic frequency of $10.385 \mathrm{MHz}$ [4]. We, however, invented a new synchronization method for the two rf's. A very important clue to solving the problem was the fact that the electron beam from a linac is generally not continuous but pulsing. We therefore thought that an rf for a linac might be fed only in a short time during beam emission and acceleration. This idea is completely different from the general method: an rf continuously generated by a synthesizer. An electron gun trigger signal at SPring-8 is generated by using the $508.58 \mathrm{MHz}$ rf of the storage ring. The gun trigger signal is divided in two. One is used as a pretrigger signal for klystron modulators and the other is a gun trigger signal. In the new synchronization method, the pretrigger signal triggers a $2856 \mathrm{MHz}$ rf generator, which consists of an arbitrary waveform generator (AWG) and a frequency multiplier. The time width generating the $2856 \mathrm{MHz}$ rf is about $290 \mu \mathrm{s}$. With this method, two rf's are automatically synchronized.

In this Letter, we report on a new synchronization method of two rf's by means of a novel technique. The rf for a linac is generated by the rf of a circular accelerator. The uniqueness of this method is that an rf for a linac is not continuously generated but pulsing. The rf generator apparatus for a linac is simple and can be applied to any combination of two rf's. With this new synchronization method at SPring-8, beam intensity from the linac was kept almost constant even with higher peak current, and 
the shift of the beam energy center became smaller than that when a synthesizer is used.

We explain the new method of synchronizing the two rf's by presenting a concrete example at SPring- 8 . The apparatus consists of two components: an arbitrary waveform generator and a frequency multiplier. With the use of this apparatus, we show how to make the $2856 \mathrm{MHz}$ rf for the linac by using $508.58 \mathrm{MHz}$ rf for the storage ring. This method can also be applied to general cases. We assume that value $f 1$ equals $508.58 \mathrm{MHz}$ divided by an arbitrary positive integer $N . f 1$ is expressed by

$$
f 1=508.58 \mathrm{MHz} / N .
$$

The value obtained is multiplied by an arbitrary positive integer $M$, giving the following equation:

$$
\begin{aligned}
f 2 & =f 1 \times M \\
& =f 1 \times(M 1 \times M 2) \\
& =(508.58 \mathrm{MHz} \times M 1 / N) \times M 2,
\end{aligned}
$$

where $M 1$ and $M 2$ take on positive integers. Moreover, $M 2$ must be an even number. The reason will be explained later. The best value of $f 2$ is equal to the $\mathrm{rf}$ of the linac. The difference between $f 2$ and the true $2856 \mathrm{MHz}$ rf is presented by $f 3$, where

$$
f 3=2856 \mathrm{MHz}-f 2 .
$$

With Eqs. (1) and (2), we can obtain a frequency $f 4$, which should be generated by an arbitrary waveform generator. $f 4$ can be written as

$$
f 4=f 1 \times M 1=(508.58 \mathrm{MHz} / N) \times M 1 .
$$

A frequency multiplier can produce only a frequency multiplied by an even number. When we define $M 2$ as a multiplication factor for the frequency multiplier, $M 2$ can take only an even number. Since the value of $M 2$ is limited, the value of $M 1$ is also automatically limited. As an example, we look for the values of $N$ and $M$ in the region of 1-10000 using a personal computer so as to minimize the value of $f 3$, and obtain $N=921$ and $M=5172$. In this case, the minimum value of $f 3$ is $244 \mathrm{~Hz}$. $M$ is rewritten with even numbers as

$$
M=5172=2586 \times 2=1293 \times 4 .
$$

As the multiplication factor of $M 2$, we take the number 4 in Eq. (5). The value of $M 1$ automatically becomes 1293. The frequency generated by the arbitrary waveform generator is given by Eq. (4) as

$$
\begin{aligned}
f 4 & =f 1 \times M 1 \\
& =(508.58 \mathrm{MHz} / 921) \times 1293 \\
& =713.999 \mathrm{MHz} .
\end{aligned}
$$

The frequency obtained is larger than the $508.58 \mathrm{MHz} \mathrm{rf}$, so it is not worthy to adopt. This example indicates that we cannot simply select the values of $N$ and $M$ to minimize the value of $f 3$. Thus, in selecting $N$ and $M$, we must set physical restrictions. The selection rules for $N, M 1$, and $M 2$ are as follows: (i) it is better for the value of $f 3$ to be minimized; (ii) since $M 2$ is a multiplication factor for a frequency multiplier, a small value is preferable (we set the maximum value of $M 2$ at 40); and (iii) a frequency generated by an arbitrary waveform generator must be smaller than the rf for a circular accelerator so that a narrow bandpass filter can be installed in the output signal from the arbitrary waveform generator. In our case, we first chose a multiplication factor for the frequency multiplier and fixed the factor of 32. Under this condition, we looked for the minimum value of $f 3$ for selecting $N$ and $M 1$ and obtained $N=359$ and $M 1=63$, respectively. With these values, we can derive the frequency $f 4$ generated by the arbitrary waveform generator as

$$
f 4=(508.58 \mathrm{MHz} / 359) \times 63=89.249415 \mathrm{MHz} .
$$

Passing the $f 4$ through the frequency multiplier, the frequency is converted to

$$
\begin{aligned}
f 2 & =f 4 \times M 2 \\
& =89.249415 \mathrm{MHz} \times 32 \\
& =2855.981281 \mathrm{MHz} .
\end{aligned}
$$

It is clear that the frequency obtained is synchronous with the $\mathrm{rf}$ of the storage ring. In the case of SPring- 8 , we show in the block diagram in Fig. 1 that the $2856 \mathrm{MHz}$ rf of the linac is generated by the $508.58 \mathrm{MHz}$ rf of the storage ring. In the figure, the rf of the storage ring as an external clock and the gun trigger signal as a start signal at the rate of $60 \mathrm{~Hz}$ are supplied to the arbitrary waveform generator. Since the arbitrary waveform generator is generally available as a commercialized product, we chose AWGs produced by SONY-Techtronix: the AWG2041 (8 bit) and AWG500 (10 bit). These AWGs are equipped with external clock and start timing signal inputs. We wrote a program to generate only an arbitrary waveform. There are many sideband frequencies around 89.249415 MHz. In order to reject them around the main frequency, we inserted a very narrow bandpass filter for $89.249415 \mathrm{MHz}$. The bandwidth is $89.25 \pm 3 \mathrm{MHz}$. If no narrow bandpass filter is inserted, we can observe many large sideband frequencies around the $2855.981281 \mathrm{MHz}$ by using a spectrum analyzer. These sideband frequencies cause large shifts in the center of electron beam energy. The amplitude level of the $2855.981281 \mathrm{MHz}$ rf attains a normal level very slowly at about $70 \mu$ s because of the narrow bandpass filter. Thus, we set it so that the $89.249415 \mathrm{MHz}$ generated by the AWG is generated for a short duration of about $290 \mu \mathrm{s}$. We also found that a narrow bandpass filter for the $508.58 \mathrm{MHz}$ rf supplied to the AWG suppressed the sideband frequencies at around 2855.981281 MHz. We therefore inserted a narrow bandpass filter of $508.58 \pm 1 \mathrm{MHz}$. The frequency of the $2855.981281 \mathrm{MHz}$ rf generated by 


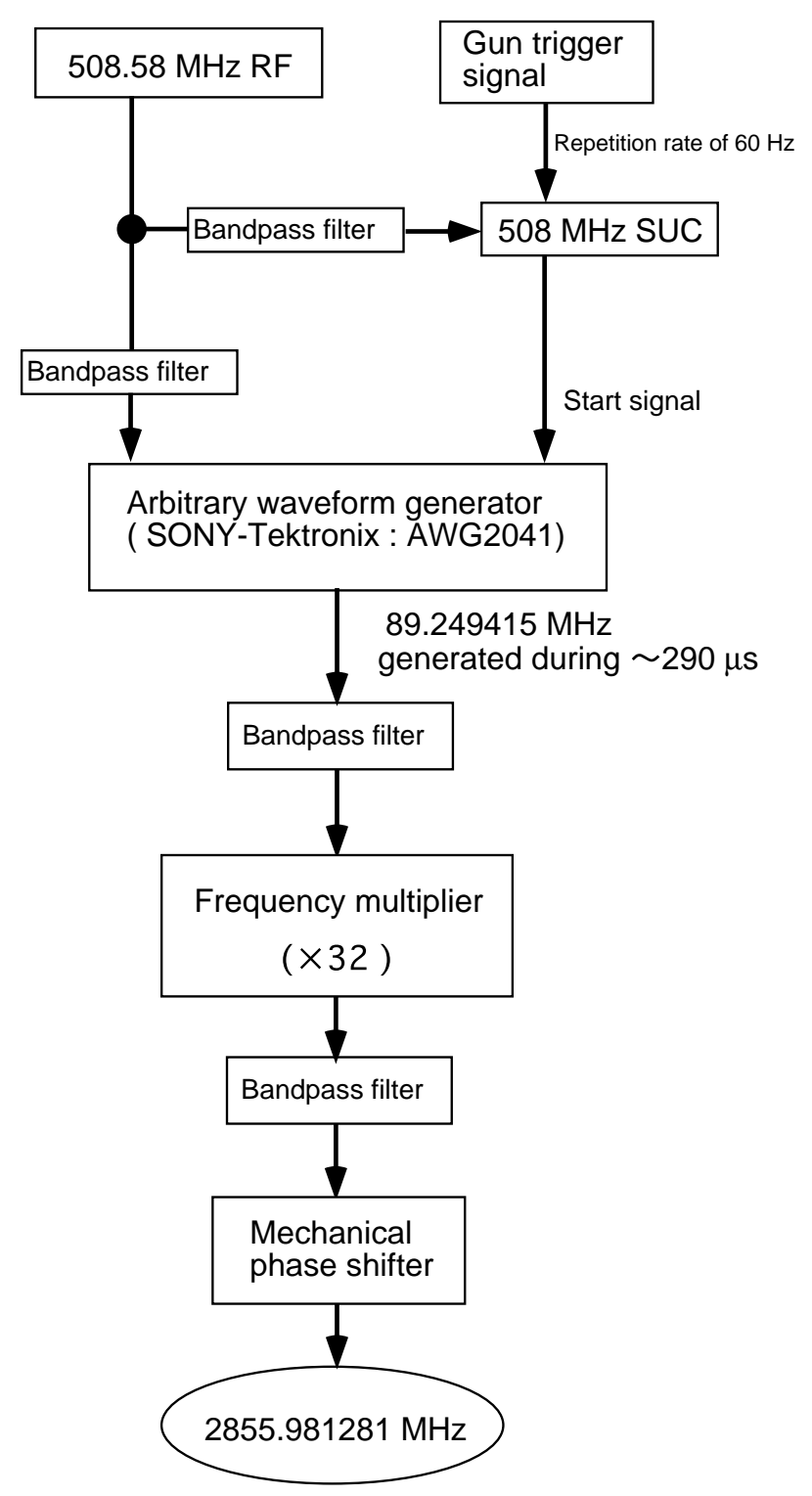

FIG. 1. Block diagram of the new synchronization method between the 508.58 and $2856 \mathrm{MHz}$ rf's. The system consists of an arbitrary waveform generator and a frequency multiplier. The $2856 \mathrm{MHz}$ rf is generated only for a short duration of about $290 \mu \mathrm{s}$.

the new method, as shown in Fig. 1, was analyzed by a spectrum analyzer. The spectra obtained is shown in Fig. 2, where the frequency is exactly the same as Eq. (8). The $2856 \mathrm{MHz}$ rf generated by a synthesizer (Hewlett Packard HP8664A), which is operated at present, is also shown in Fig. 2. The difference of $-18.719 \mathrm{kHz}$ between the two $2856 \mathrm{MHz}$ rf's can be seen, but this difference is too small to have any negative effect on the electron beam, because acceleration cavities, in general, have a $Q$ value ( $Q=f / \Delta f$, where $f$ is the resonant frequency of a cavity and $\Delta f$ is the full width at half maximum). In the case of the linac at SPring- 8 , for example, the $Q$ value and $\Delta f$ are 13500 and $211.6 \mathrm{kHz}$, respectively. The difference of

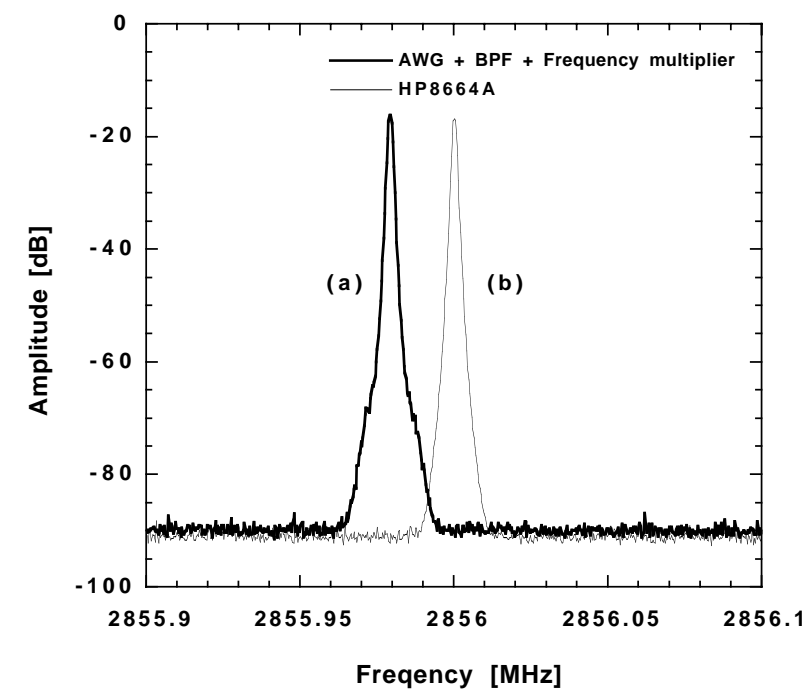

FIG. 2. Obtained spectra of $2856 \mathrm{MHz}$ rf's: (a) generated by the new method and (b) generated by a synthesizer.

$-18.719 \mathrm{kHz}$ is small compared with $211.6 \mathrm{kHz}$ and is not crucial in an actual beam operation. To be certain that the effect is small, we carried out the linac beam study by changing the fundamental rf of $2856 \mathrm{MHz}$ from 2855.080 to $2856 \mathrm{MHz}$ generated by the synthesizer. The center of electron beam energy shifted around $0.2 \%$. A correction can easily be made by changing the high voltage of power equipments for klystrons. As long as the difference between the two rf's is smaller than the $\Delta f$ of a cavity used, no serious problem will occur in an actual beam operation.

With the new synchronization method of the two rf's, tests and optimization of the beam operation were conducted. The block diagram for the study is shown in Fig. 3. In Fig. 3, the gun trigger signal is divided in two. One enters into the AWG that generates $89.249415 \mathrm{MHz}$. After passing through the 32-time frequency multiplier, the $2855.981281 \mathrm{MHz}$ rf of the linac is generated for a short duration of about $290 \mu \mathrm{s}$. The rf is transmitted approximately $150 \mathrm{~m}$ through a phase-stabilized optic fiber [5]. The transmitted $2855.981281 \mathrm{MHz}$ rf is connected to an rf switch, which can remotely select the continuously generated $2856 \mathrm{MHz}$ rf or the newly generated $2856 \mathrm{MHz}$ rf. The other gun trigger signal is arbitrarily delayed by a $508 \mathrm{MHz}$ synchronous universal counter (508 MHz SUC) [6] and transmitted to the gun high-voltage deck through a phase-stabilized optic fiber, where the signal triggers a grid pulser [2]. A pulse signal transmission system with small time jitters is used [6].

The total timing chart, based on the block diagram shown in Fig. 3, is presented in Fig. 4. It should be noted that all signals are synchronized with the $508.58 \mathrm{MHz}$ rf. In Fig. 4, the center of the electron beam must be correctly injected into the zero-crossing point of the 2855.981281 MHz rf in the prebuncher cavity. Watching 


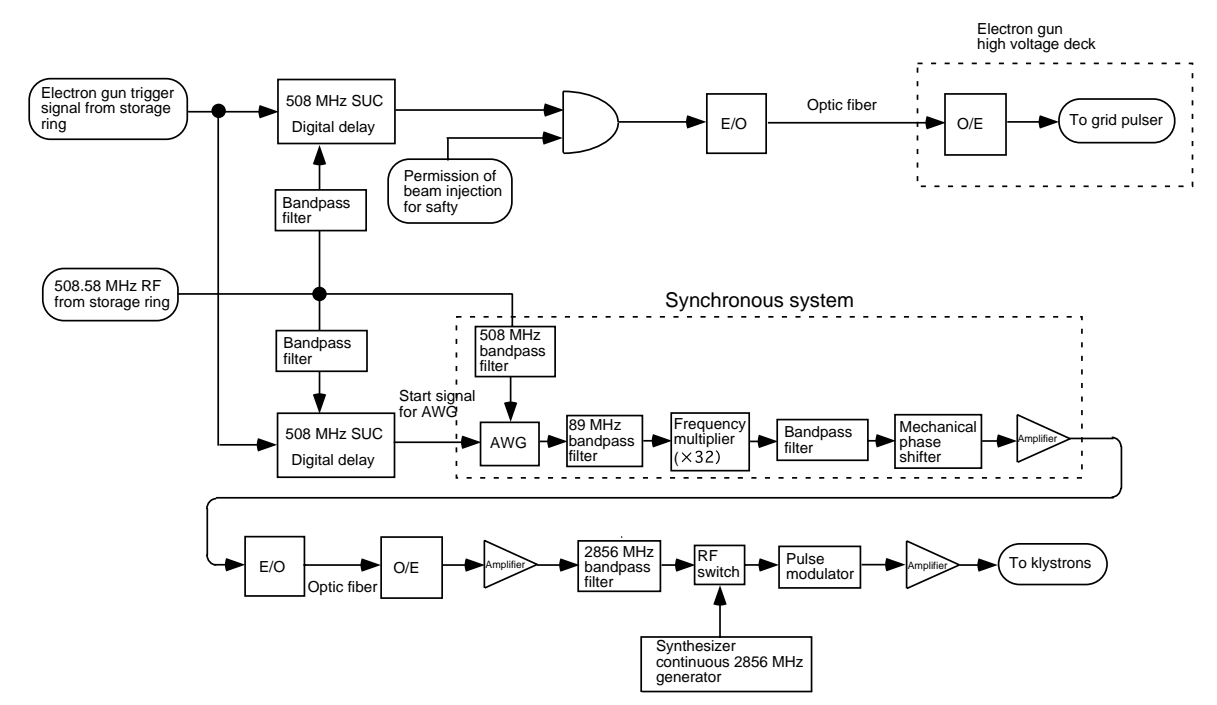

FIG. 3. Test scheme of the new synchronization method for electron beam operation. E/O and O/E are an electric-to-optic converter and an optic-to-electric converter, respectively. An electron gun trigger signal and 508.58 MHz rf generated in the storage ring are sent to the linac control room through phase-stabilized optic fibers. To compare the original continuous waveform of the $2856 \mathrm{MHz}$ rf generated by a synthesizer with the $2856 \mathrm{MHz}$ rf generated by the new method, an rf switch to select one of them is inserted before the pulse modulator. All apparatuses are synchronized with the $508.58 \mathrm{MHz}$ rf for the storage ring.

beam current monitors installed in the exits of the gun high-voltage deck and the main buncher, respectively, the phase of the $2855.981281 \mathrm{MHz}$ rf subtly tuned the electron beam injection timing with a mechanical phase shifter as shown in Fig. 3 to achieve maximum beam transmission efficiency. After completing tuning, we measured parameters of the electron beam in the linac. The measurement procedure is as follows: First, We set the grid pulser at a pulse width of 250 ps FWHM. In the nonsynchronization method, we observed a dizzy change of the beam intensity at the energy of $1 \mathrm{GeV}$ shot by shot. On the other hand, in the case of the new synchronization method, the beam intensity was kept constant, as we expected. Next, we set a pulse width of $1 \mathrm{~ns}$. We increased the peak current in the linac from $200 \mathrm{~mA}$ to $1.8 \mathrm{~A}$. The beam energy stability was observed to change from $0.018 \%$ to $0.030 \%$ as a standard deviation for the nonsynchronization method. For the synchronization method, however, the energy stability was not only almost constant, but also fell to $0.015 \%$, a value which was smaller. Thus, beam quality was remarkably improved with the new synchronization method.

The merits of the new synchronization method of arbitrary different rf's are summarized as follows: This method is applicable to any electron beam facility consisting of linac and circular accelerators. The choice of rf's, which had been previously limited by klystrons, becomes flexible. The rf for a linac can be easily generated by using the rf of a circular accelerator, as shown in Fig. 1. The apparatus to generate an rf for a linac consists of an arbitrary waveform generator and a frequency multiplier. The method itself is very simple and can be easily constructed. Construction simply requires making a frequency

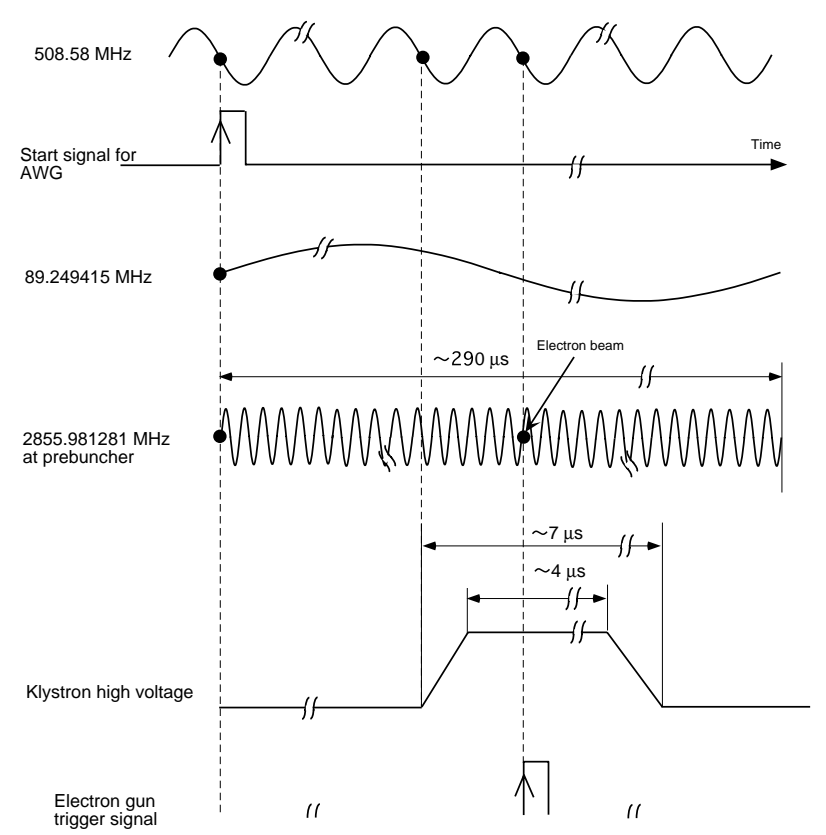

FIG. 4. Schematic timing charts for all signals, that is, a $508.58 \mathrm{MHz}$ rf, start signal for an AWG, generated $2856 \mathrm{MHz}$ rf, supplied high voltage for klystrons, and electron gun trigger signal. In particular, the start signal for the AWG, high voltage supplied to the klystrons, and the gun trigger signal are synchronized with the $508.58 \mathrm{MHz}$ rf. Start time of the $2856 \mathrm{MHz}$ rf is guaranteed by synchronization with the $508.58 \mathrm{MHz}$ rf. Phase differences in any time points between the two rf's are kept constant for approximately $290 \mu \mathrm{s}$. 
multiplier and inputting the data information of an rf into an AWG. The phase between the two rf's is automatically synchronized by the new method.

\section{ACKNOWLEDGMENTS}

The authors thank Dr. H. Ego, Dr. Y. Ohashi, Dr. T. Ohshima, and the staff of the linac and the booster synchrotron for their help. We also thank Mrs. T. Hori and T. Kobayashi for their indispensable assistance in the operation of the linac. In addition, the authors are grateful to Mr. K. Jitsuno of SONY-Techtronix for manufacturing a 10-bit AWG. Thanks are due especially to Dr. N. Kumagai for his support.
[1] M. Hara et al., in Proceedings of the 2nd European Particle Accelerator Conference, Nice (Institute of Physics, Bristol, UK, 1990), Vol. 1, pp. 466-468.

[2] T. Kobayashi et al., in Proceedings of the XIX International Linac Conference, Chicago (ANL Report No. ANL-98/28, 1998), p. 58-60.

[3] S. Suzuki et al., in Proceedings of the 1993 Particle Accelerator Conference, Washington DC (IEEE, Piscataway, NJ, 1993), Vol. 1, pp. 602-604.

[4] E. Ezura et al., KEK Report No. 98-93, 1998.

[5] J. Urakawa, Part. Accel. 29, 251 (1990); KEK Report No. 90-5, 1990.

[6] H. Suzuki et al., Nucl. Instrum. Methods Phys. Res., Sec. A 431, 294-305 (1999). 